\title{
Primary pyomyositis in North India: a clinical, microbiological, and outcome study
}

\author{
Susheel Kumar ${ }^{1}$, Ashish Bhalla ${ }^{1}$, Rajveer Singh ${ }^{1}$, Navneet Sharma ${ }^{1}$, Aman Sharma ${ }^{1}$, Vikas Gautam ${ }^{2}$, \\ Surjit Singh ${ }^{1}$, and Subhash Varma ${ }^{1}$
}

Departments of ${ }^{1}$ Internal Medicine and ${ }^{2}$ Medical Microbiology, Postgraduate Institute of Medical Education and Research, Chandigarh, India
Received: December 24, 2015

Revised : June 20, 2016

Accepted: July 13, 2016

\section{Correspondence to}

Susheel Kumar, M.D.

Department of Internal Medicine, Postgraduate Institute of Medical Education and Research, Chandigarh 160-012, India

Tel: +91-977-9178384

Fax: +91-172-2744401

E-mail:sukubhu@outlook.com
Background/Aims: Pyomyositis is an infective condition with primary involvement of the skeletal muscles. There is sparse recent literature on patients with pyomyositis.

Methods: This study was carried out at emergency services of a tertiary care center located in subtropical area of Indian subcontinent.

Results: Sixty-two patients of primary pyomyositis formed the study cohort. Mean age of occurrence was $29.9 \pm 14.8$ years. There were 54 men. Twelve patients had underlying medical diseases. Muscle pain was seen in all 62 patients. Forty-eight patients (77.4\%) had the fever. Most common site of involvement was thigh muscles $(n=29,46.8 \%)$. Forty-nine patients (79\%) presented in the suppurative stage of illness. Patients with comorbidities were older (age: median 36 years [interquartile range (IQR), 25 to 47] vs. 24 years [IQR, 16 to 35], $p=0.024$ ), had higher culture positivity with gram-negative organisms (8/9 [88.89\%] vs. 6/29 [20.69\%], $p=0.001)$. Importantly, higher number of these patients received inappropriate antibiotics initially. Patients with positive pus culture result had higher complication rate $(32 / 38$ [84.21\%] vs. $10 / 18$ [55.56\%], $p=0.044)$. Six patients $(9.7 \%)$ had in-hospital mortality. Lower first-day serum albumin, initial inappropriate antibiotic therapy, and advanced form of the disease at presentation were associated with increased in-hospital mortality.

Conclusions: Primary pyomyositis is not an uncommon disease entity. Patients with comorbidities were more likely to receive initial inappropriate antibiotic therapy. Patients with positive pus culture report had the higher rate of complications. Lower first-day serum albumin, initial inappropriate antibiotic therapy and advanced form of the disease at presentation were associated with increased in-hospital mortality.

Keywords: Primary pyomyositis; Outcome; Emergency services

\section{INTRODUCTION}

Pyomyositis is an infective condition with primary involvement of the skeletal muscles manifesting mainly as single or multiple intramuscular abscesses. It was originally reported from tropical regions of Asia and
Africa. This disease entity is now being increasingly reported from temperate areas as well especially in patients with comorbid conditions [1-14]. It is most common in young age group, and various case series showed the male predominance. This disease entity progresses through three distinct clinical stages: stage 1 or the in- 
vasive stage, stage 2 or the suppurative stage of illness followed by late stage 3 or disseminated form of the disease. The majority of patients are seen during the suppurative stage of illness. Staphylococcus aureus is the most common organism isolated from these patients [1-3]. The exact pathogenesis is not fully elucidated yet. It has been though postulated that transient bacteremia in the setting of pre-existing or concurrent muscular injury leads to implantation and growth of bacteria leading to pyomyositis [1-3]. It's relative unfamiliarity, non-specific clinical symptoms, and subtle local signs initially lead to the delay in diagnosis resulting in increased morbidity and mortality. There is also paucity of recent literature on this disease entity. Therefore, this study was planned to look at the spectrum of clinical presentations, underlying medical illness, the course in hospital, complications developed and hospital outcome in these patients presenting at emergency services of a tertiary care center located in subtropical area of Indian subcontinent.

\section{METHODS}

This study was carried out at emergency services of Postgraduate Institute of Medical Education and Research, Chandigarh, a tertiary care referral hospital in Northern India. More than 75,000 patients visit emergency of this institute annually. We reviewed clinical records of all patients of primary pyomyositis presenting in emergency services from July 2007 onwards, and relevant clinical details were recorded in a predesigned instrument. From July 2007 to December 2012, records of patients were obtained from medical record library, whereas patients were recruited prospectively from January 2013 till December 2013. The medical record library uses the ICD-10 (International Statistical Classification of Diseases and Related Health Problems 10) system for classification of diseases. Each disease condition is assigned a unique code number. We retrieved medical records of primary pyomyositis patients using the specific code number assigned to this disease entity and recorded data of those patients of primary pyomyositis presenting in emergency services. Primary pyomyositis was defined as an intramuscular infection involving one or more of the skeletal muscle groups in the absence of adjacent skin, soft tissue, or bone infection [1-3]. Age, sex, history of muscle trauma, duration of illness, presenting manifestations, comorbid conditions, hospital course, treatment instituted, complications encountered, and hospital outcome of these patients were recorded as well. Investigations including complete blood count, differential blood count, erythrocyte sedimentation rate (ESR), liver and renal function tests, gram stain and culture of the pus from muscles, acid-fast bacilli (AFB) stain/culture and blood culture reports were also recorded. Radiological investigations including chest $\mathrm{X}$-ray, X-ray dorsolumbar spine, 2D Echo, ultrasonography, computed tomography (CT), magnetic resonance imaging (MRI) reports wherever available/done were recorded in a predesigned instrument. The Institution Ethics Committee approved the study. Informed consent was obtained from patients who were recruited prospectively from January 2013 till December 2013.

Sepsis was defined as the presence of a clinically identified site of infection and two or more of the following: temperature $>38^{\circ} \mathrm{C}$ or $<36^{\circ} \mathrm{C}$; heart rate $>90$ beats per minute; respiratory rate $>20$ breaths per minute or $\mathrm{PaCO}_{2}<32 \mathrm{mmHg}$; and white blood cell count $>12,000 /$ $\mu \mathrm{L},<4,000 / \mu \mathrm{L}$, or $>10 \%$ immature forms (i.e., bands), altered mental status (Glasgow coma scale $[$ GCS $]<9$ ), significant edema or positive fluid balance $(>20 \mathrm{~mL} / \mathrm{kg}$ over 24 hours), hyperglycemia (glucose $>120 \mathrm{mg} / \mathrm{dL}$ ) in the absence of diabetes [14]. Various laboratory abnormalities were defined using cut-off values as follows: anemia (hemoglobin < $13 \mathrm{~g} / \mathrm{dL}$ for male, < $12 \mathrm{~g} / \mathrm{dL}$ for female, non-pregnant); leukocytosis (total leukocyte count [TLC] $>12,000 / \mu \mathrm{L})$, leucopenia $(\mathrm{TLC}<4,000 / \mu \mathrm{L})$; elevated ESR ( $>20$ in the first hour); hypoalbuminemia (albumin $<3.5$ $\mathrm{g} / \mathrm{dL}$ ); and impaired renal function (blood urea nitrogen $>50 \mathrm{mg} / \mathrm{dL}$ and/or serum creatinine $>1.20 \mathrm{mg} / \mathrm{dL}$ ).

Statistical analysis was done using the statistical software SPSS version 20.0 (IBM Co., Armonk, NY, USA). Data was presented as mean \pm standard deviation $(\mathrm{SD})$ when distributed normally and as median with interquartile range (IQR), if the distribution was skewed. We divided study group into two subgroups of survivors and non-survivors and various parameters between these two subgroups were compared to identify the parameters associated with in-hospital mortality. We also compared variables among subgroups of patients with positive and negative pus culture report; patients with gram-negative and positive bacterial growth in pus; patients with 
methicillin-sensitive Staphylococcus aureus (MSSA) and methicillin-resistant Staphylococcus aureus (MRSA) infection; and patients with or without underlying medical illness. Continuous, normally distributed variables were compared with Student $t$ test, and for non-normally distributed continuous variables, non-parametric test like, Mann-Whitney $U$ test was used. Categorical variables were compared by Fisher exact test. We also compared various variables among patients with different stages of illness at presentation. For normally distributed variables between three groups, analysis of variance test was used. For non-normally distributed variables, Kruskal-Wallis test was used. $p<0.05$ were considered significant. All statistical tests performed were 2-tailed.

\section{RESULTS}

\section{Demographic, clinical, and laboratory features}

During the study period (July 2007 to December 2013), 62 patients fulfilling diagnostic criteria for primary pyomyositis were identified and formed the study cohort. The mean age of occurrence was $29.9 \pm 14.8$ years (range, 13 to 67$)$. There were 54 men and eight women. Overall, 12 patients (19.35\%) had underlying medical diseases. Among the cohort of 62 patients, 16 patients (25.8\%) gave the history of trauma to affected muscles.

Main symptom was muscle pain (myalgia), seen in all 62 patients (100\%). Local muscle pain was noticed in 54 $(87.1 \%)$ and generalized muscle pain in rest eight patients (12.9\%). Forty-eight patients (77.4\%) had the fever as one of the presenting symptoms. Local muscle swelling was noted in 47 (75.8\%), local redness in $23(22.58 \%)$, and back pain in 14 patients (22.6\%). The median time between the onset of the first symptom and diagnosis of primary pyomyositis was 10 days (IQR, 2 to 80). The most common site of involvement was thigh muscles ( $\mathrm{n}=29,46.8 \%$ ), followed by iliopsoas in 25 (40.3\%) and upper extremities in 12 (19.4\%). The relevant demographic, clinical characteristics, and laboratory features details at presentation are shown in Table 1. Anemia was present in 55 patients $(88.71 \%)$ and mean hemoglobin in these patients was $9.39 \pm 1.91 \mathrm{~g} / \mathrm{dL}$ (range, 5 to 13). Forty patients (64.52\%) had leukocytosis with mean TLC being $17,609.38 \pm 7,457.89 / \mu \mathrm{L}$ (range, 12,000 to 45,000 ) and raised ESR was present in 60 patients $(96.8 \%)$ with mean
Table 1. Demographic data, clinical characteristics, and laboratory features at initial presentation of 62 pyomyositis patients

\begin{tabular}{|c|c|}
\hline Characteristic & Value \\
\hline Age, yr & $29.9 \pm 14.8$ \\
\hline Male sex & $54(87.1)$ \\
\hline History of trauma & $16(25.8)$ \\
\hline \multicolumn{2}{|l|}{ Underlying medical disorders } \\
\hline Diabetes mellitus & $6(9.7)$ \\
\hline Chronic liver disease & $3(4.8)$ \\
\hline Malignancy & $3(4.8)$ \\
\hline \multicolumn{2}{|l|}{ Location } \\
\hline Thigh & $29(46.8)$ \\
\hline Iliopsoas & $25(40.3)$ \\
\hline Upper extremity & $12(19 \cdot 4)$ \\
\hline Chest wall & $8(12.9)$ \\
\hline Gluteal & $4(6.5)$ \\
\hline \multicolumn{2}{|l|}{ Clinical features } \\
\hline Muscle pain & $62(100)$ \\
\hline Fever & $48(77 \cdot 4)$ \\
\hline Local swelling & $47(75.8)$ \\
\hline Local redness & $23(22.6)$ \\
\hline Back pain & $14(22.6)$ \\
\hline \multicolumn{2}{|l|}{ Laboratory parameter (first day) } \\
\hline Hemoglobin, g/dL & $9.64 \pm 2.11$ \\
\hline Total leucocyte count, $/ \mu \mathrm{L}$ & $12,834 \pm 7,545 \cdot 48$ \\
\hline $\mathrm{ESR}, \mathrm{mm} / \mathrm{hr}$ & $52.50 \pm 15.38$ \\
\hline Total serum protein, g/dL & $6.49 \pm 1.25$ \\
\hline Serum albumin, g/dL & $2.71 \pm 0.74$ \\
\hline \multicolumn{2}{|l|}{ Stage at presentation } \\
\hline First stage & $6(9.7)$ \\
\hline Suppurative stage & $49(79)$ \\
\hline Late stage & $7(11.3)$ \\
\hline \multicolumn{2}{|l|}{ Duration of symptoms, day } \\
\hline Muscle pain & $10(2-80)$ \\
\hline Fever & $10(5-20)$ \\
\hline
\end{tabular}

Values are presented as mean $\pm \mathrm{SD}$, number (\%), or median (interquartile range).

ESR, erythrocyte sedimentation rate.

being $53.23 \pm 14.67 \mathrm{~mm} / \mathrm{hr}$. Hypoproteinemia was documented in 47 patients at presentation with mean total protein being $5.72 \pm 0.65 \mathrm{~g} / \mathrm{dL}$ (range, 4.3 to 6.5 ). Fifty-six 
Table 2. Demographic data, clinical characteristics, laboratory features, hospital stay, and outcome according to stage of presentation in 62 pyomyositis patients

\begin{tabular}{|c|c|c|c|c|}
\hline Characteristic & First stage $(n=6)$ & Second stage $(n=49)$ & Disseminated or late stage $(n=7)$ & $p$ value \\
\hline Age, yr & $31.67 \pm 12.99$ & $29.49 \pm 14.82$ & $31.29 \pm 18.02$ & 0.915 \\
\hline Sex & & & & 0.296 \\
\hline Male & $6(100)$ & $43(87.76)$ & $5(71.43)$ & \\
\hline Female & 0 & $6(12.24)$ & $2(28.57)$ & \\
\hline History of trauma & $2(33 \cdot 3)$ & $11(22.45)$ & $3(42.85)$ & 0.067 \\
\hline Underlying medical conditions & 0 & $10(20.40)$ & $2(28.60)$ & 0.395 \\
\hline \multicolumn{5}{|l|}{ Location } \\
\hline Gluteal & 1 & 2 & 1 & - \\
\hline Thigh & 3 & 22 & 4 & - \\
\hline Calf & - & 3 & - & - \\
\hline Upper extremity & 2 & 8 & 2 & - \\
\hline Iliopsoas & 1 & 21 & 3 & - \\
\hline Chest wall & 1 & 6 & 1 & - \\
\hline \multicolumn{5}{|l|}{ Clinical feature } \\
\hline Muscle pain & $6(100)$ & $49(100)$ & $7(100)$ & - \\
\hline Fever & $4(66.67)$ & $37(75 \cdot 5)$ & $7(100)$ & 0.281 \\
\hline Back pain & $2(33 \cdot 33)$ & $10(20.41)$ & $2(28.57)$ & 0.769 \\
\hline Swelling & $4(66.67)$ & $37(75 \cdot 51)$ & $6(85.71)$ & 0.912 \\
\hline Redness & $2(33 \cdot 33)$ & $17(34.69)$ & $4(57.14)$ & 0.867 \\
\hline Fever, day & $14.20 \pm 10.64$ & $14.16 \pm 13.71$ & $15 \cdot 57 \pm 8.41$ & 0.965 \\
\hline Muscle swelling, day & $9.00 \pm 6.52$ & $19.16 \pm 11.10$ & $13.80 \pm 4.15$ & 0.718 \\
\hline Pulse rate, /min & $93.33 \pm 11.43$ & $93.14 \pm 7.65$ & $96.86 \pm 19.92$ & 0.820 \\
\hline $\mathrm{SBP}, \mathrm{mmHg}$ & $116.23 \pm 4.97$ & $120.78 \pm 19.38$ & $100.86 \pm 6.20$ & $0.025^{\mathrm{a}}$ \\
\hline $\mathrm{DBP}, \mathrm{mmHg}$ & $75 \pm 5 \cdot 48$ & $77.80 \pm 9.76$ & $68.57 \pm 3.78$ & $0.044^{\mathrm{a}}$ \\
\hline Respiratory rate, /min & $18.33 \pm 2.33$ & $17 \cdot 33 \pm 4 \cdot 32$ & $21.02 \pm 6.39$ & 0.253 \\
\hline GCS & 15 & 15 & $13.14 \pm 3.19$ & $<0.001^{b}$ \\
\hline \multicolumn{5}{|l|}{ Laboratory parameter (first day) } \\
\hline Hemoglobin, g/dL & $10.03 \pm 2.50$ & $9.41 \pm 2.06$ & $10.68 \pm 2.01$ & 0.977 \\
\hline $\mathrm{TLC}, / \mu \mathrm{L}$ & $9,750 \pm 5,516.43$ & $10,385 \cdot 71 \pm 6,215 \cdot 9$ & $13,577.08 \pm 7,863.9$ & 0.338 \\
\hline $\mathrm{ESR}, \mathrm{mm} / \mathrm{hr}$ & $37 \cdot 50 \pm 18.52$ & $48.71 \pm 6.89$ & $54.84 \pm 15.45$ & 0.076 \\
\hline Serum urea, mg/dL & $62.33 \pm 53.16$ & $49.25 \pm 44.09$ & $80.00 \pm 67.49$ & 0.265 \\
\hline Serum creatinine, $\mathrm{mg} / \mathrm{dL}$ & $1.17 \pm 0.84$ & $0.99 \pm 0.77$ & $1.33 \pm 0.67$ & 0.541 \\
\hline Total protein, g/dL & $7.63 \pm 1.34$ & $6.47 \pm 1.20$ & $5.73 \pm 1.07$ & $0.039^{c}$ \\
\hline Serum albumin, g/dL & $2.82 \pm 0.45$ & $2.74 \pm 0.81$ & $2.40 \pm 0.32$ & 0.550 \\
\hline SGOT, U/L & $71.80 \pm 48.27$ & $67.00 \pm 59.42$ & $56.67 \pm 16.56$ & 0.935 \\
\hline SGPT, U/L & $71.80 \pm 80.26$ & $54 \cdot 78 \pm 44 \cdot 32$ & $68.00 \pm 24.44$ & 0.822 \\
\hline Bilirubin, total, mg/dL & $0.87 \pm 0.52$ & $0.93 \pm 0.83$ & $0.68 \pm 0.22$ & 0.804 \\
\hline Positive pus culture & - & $32 / 49(65 \cdot 3)$ & $6 / 7(85.7)$ & 0.003 \\
\hline Positive blood culture & o & $5 / 49(10.20)$ & $3 / 7(42.85)$ & 0.002 \\
\hline \multicolumn{5}{|l|}{ Management } \\
\hline Percutaneous & - & $16(32.65)$ & $2(28.57)$ & 0.438 \\
\hline
\end{tabular}


Table 2. Continued

\begin{tabular}{lcccc}
\hline Characteristic & First stage $(\mathrm{n}=6)$ & Second stage $(\mathrm{n}=49)$ & Disseminated or late stage $(\mathrm{n}=7)$ & $p$ value \\
\hline Surgical drainage & - & $33(67.35)$ & $5(71.43)$ & 0.611 \\
Appropriateness of antibiotic & - & $20 / 32(62.5)$ & $5 / 6(83.3)$ & 0.643 \\
Hospital stay, day & $11.00 \pm 6.69$ & $14.94 \pm 9.99$ & $8.71 \pm 7.67$ & $4 / 7(57.14)$ \\
In-hospital mortality & 0 & $2 / 49(4.08)$ & $<.379$ & $<.001$ \\
\hline
\end{tabular}

Values are presented as mean $\pm \mathrm{SD}$ or number $(\%)$.

SBP, systolic blood pressure; DBP, diastolic blood pressure; GCS, Glasgow coma scale; TLC, total leucocyte count; ESR, erythrocyte sedimentation rate; SGOT, serum glutamic oxaloacetic transaminase; SGPT, serum glutamic pyruvic transaminase.

${ }^{\mathrm{a}} \mathrm{p}<0.05$ between second and late stage.

${ }^{\mathrm{b}} p<0.05$ between first, second, and late stage.

${ }^{c} p<0.05$ between first and late stage.

patients (90.3\%) had hypoalbuminemia at presentation and mean albumin was $2.62 \pm 0.57 \mathrm{~g} / \mathrm{dL}$ (range, 1.8 to 3.5).

Forty-nine patients $(79 \%)$ presented in suppurative stage (stage 2), seven patients (11.3\%) in late-stage (stage 3) while rest six patients (9.7\%) presented in invasive stage (stage 1) of illness. We compared demographic characteristics, clinical presentation findings, various laboratory parameters including culture results for pus or blood, appropriateness of initial antibiotics, complications encountered, methods of drainage of pus, duration of hospital stay, and hospital outcome among patients classified on the basis of stage of presentation. On univariate comparison, we found patients with the disseminated stage of infection had significantly worse GCS as compared to patients with either stage 1 or 2 of illness at presentation. Both systolic and diastolic blood pressure was found to be significantly lower in patients with the disseminated stage of infection as compared to patients with the suppurative/second stage of infection. Mean ESR and TLC showed a graded relationship according to the stage of presentation being highest in the stage 3 disease though these did not reach the statistical significance. Mean total serum protein was significantly lower in the disseminated stage of infection as compared to patients with the invasive stage of infection. Mean serum albumin level at admission also showed graded relationship being lowest in stage 3 of illness though it did not reach the statistical significance. In-hospital mortality was significantly higher in patients with the disseminated stage of illness. Further elaboration of the result of the comparison of various variables among different stage of illness has been done in Table 2.

Various factors were compared between patients with pyomyositis with or without underlying medical illness (Table 3). Patients with underlying medical diseases were significantly older as compared to those with no underlying medical diseases (age: median 36 years [IQR, 25 to 47 ] vs. 24 years [IQR, 16 to 35], $p=0.024$ ), Duration of fever and muscle swelling were longer in patients with underlying medical diseases, albeit these did not reach statistical significance. Patients with underlying medical diseases had significantly higher culture positivity with gram-negative organisms (8/9 [88.89\%] vs. 6/29 [20.69\%], $p=0.001$ ). Importantly, the higher number of these patients received inappropriate antibiotics initially (6/9 [66.67\%] vs. 7/29 [24.14\%], $p=0.001)$.

Various imaging methods were used for diagnosis. All patients underwent ultrasonography of involved areas. CT was performed in 15 (24.19\%) and MRI was performed in three patients (4.84\%).

\section{Microbiological investigations and etiologic agents identified}

The causative organisms for pyomyositis were primarily isolated with pus culture in 38/56 patients (67.9\%); pus was acquired either via percutaneous or surgical interventions. Gram-positive organisms were grown in 27 patients (71.05\%) while gram-negative organisms were isolated in 14 patients (36.84\%). The most common organism cultured from pus was $S$. aureus (n = $26 / 38,68.42 \%$ ). Out of 27 patients with gram-positive organisms, MSSA was isolated in 18 and MRSA in eight 
Table 3. Comparison of demographic data, clinical characteristics, laboratory features, hospital stay, and outcome among patients with or without underlying medical conditions in cohort of 62 pyomyositis patients

\begin{tabular}{|c|c|c|c|}
\hline Characteristic & $\begin{array}{l}\text { Patients with underlying medical } \\
\text { conditions }(\mathrm{n}=12)\end{array}$ & $\begin{array}{l}\text { Patients without underlying medi- } \\
\text { cal conditions }(n=50)\end{array}$ & $p$ value \\
\hline Age, yr & $36(25-47)$ & $24(16-35)$ & 0.024 \\
\hline Sex & & & 0.177 \\
\hline Male & $9(75)$ & $45(90)$ & \\
\hline Female & $3(25)$ & $5(10)$ & \\
\hline History of trauma & $4(33.33)$ & $12(24)$ & 0.478 \\
\hline \multicolumn{4}{|l|}{ Clinical features } \\
\hline Muscle pain & $12(100)$ & $50(100)$ & - \\
\hline Fever & $9(75)$ & $39(78)$ & 0.546 \\
\hline Back pain & $3(25)$ & $11(22)$ & 0.615 \\
\hline Swelling & $8(66.67)$ & $39(78)$ & 0.728 \\
\hline Redness & $6(50)$ & $17(34)$ & 0.237 \\
\hline Fever, day & $20(7-29)$ & $10(5-19)$ & 0.081 \\
\hline Muscle swelling, day & $24(6-51)$ & $10(6-15)$ & 0.072 \\
\hline Pulse rate, $/ \mathrm{min}$ & $95(81.50-103)$ & $97(85-110)$ & 0.443 \\
\hline $\mathrm{SBP}, \mathrm{mmHg}$ & $115(110-120)$ & $119(110-124)$ & 0.718 \\
\hline $\mathrm{DBP}, \mathrm{mmHg}$ & $74(70-80)$ & $80(70-80)$ & 0.895 \\
\hline Respiratory rate, /min & $18(14.50-21.50)$ & $18(16-24)$ & 0.550 \\
\hline GCS & $15(15-15)$ & $15(15-15)$ & 0.256 \\
\hline \multicolumn{4}{|l|}{ Laboratory parameter (first day) } \\
\hline Hemoglobin, g/dL & $8.80(7.15-11.63)$ & $9.60(8.30-11.10)$ & 0.297 \\
\hline $\mathrm{TLC}, / \mu \mathrm{L}$ & $11,300(7,475-16,475)$ & $11,000(8,500-17,650)$ & 0.670 \\
\hline $\mathrm{ESR}, \mathrm{mm} / \mathrm{hr}$ & $59(42-68.50)$ & $55(42-64)$ & 0.550 \\
\hline Serum urea, mg/dL & $32(24-52.25)$ & $36(20.80-82.50)$ & 0.730 \\
\hline Serum creatinine, mg/dL & $1.01(0.65-1.17)$ & $0.80(0.80-1.20)$ & 0.384 \\
\hline Total protein, g/dL & $6.35(5.25-7.55)$ & $6.68(5.50-7.58)$ & 0.742 \\
\hline Serum albumin, g/dL & $2.60(2.02-2.80)$ & $2.90(2.20-3.20)$ & 0.873 \\
\hline SGOT, U/L & $69(28.25-112.75)$ & $43.00(30.25-75.25)$ & 0.333 \\
\hline SGP'T, U/L & $51(22.25-71.50)$ & $35.50(25.25-60.50)$ & 0.484 \\
\hline Bilirubin, total, mg/dL & $0.65(0.46-0.90)$ & $0.80(0.40-1.00)$ & 0.286 \\
\hline Late stage at presentation & $2(16.67)$ & $5(10)$ & 0.395 \\
\hline Positive pus culture & $9 / 12(75)$ & $29 / 44(65.91)$ & 0.732 \\
\hline Gram-negative growth in pus & $8 / 9(88.89)$ & $6 / 29(20.69)$ & 0.001 \\
\hline Positive blood culture & $2(16.67)$ & $6(12)$ & 0.672 \\
\hline Initial inappropriate antibiotic & $6 / 9(66.67)$ & $7 / 29(24.14)$ & 0.001 \\
\hline \multicolumn{4}{|l|}{ Management } \\
\hline Percutaneous & $4(40)$ & $14(30.43)$ & 0.414 \\
\hline Surgical drainage & $6(60)$ & $32(69.57)$ & 0.576 \\
\hline Complications & $6(50)$ & $36(66.67)$ & 0.611 \\
\hline Hospital stay, day & $11.50(7-17.25)$ & $13.50(7.75-23.00)$ & 0.550 \\
\hline In-hospital mortality & $2(16.67)$ & $4(8)$ & 0.328 \\
\hline
\end{tabular}

Values are presented as median (interquartile range) or number (\%).

SBP, systolic blood pressure; DBP, diastolic blood pressure; GCS, Glasgow coma scale; TLC, total leucocyte count; ESR, erythrocyte sedimentation rate; SGOT, serum glutamic oxaloacetic transaminase; SGPT, serum glutamic pyruvic transaminase. 
patients. Enterococcus was grown in one patient. Panton-Valentine leukocidin, a cytotoxin frequently associated with community-acquired MRSA (CA-MRSA), was not tested in index study. Among 14 patients with gram-negative isolates, six patients had Escherichia coli in pus culture, three had Acinetobacter baumannii, two each grew Pseudomonas aeruginosa and Klebsiella while one patient's pus culture grew Proteus mirabilis. Out of six isolates of E. coli, four were resistant to quinolone. We do not have data on extended-spectrum $\beta$ lactamase (ESBL) production as same was not looked for. Among these patients with gram-negative isolates, five patients had type 2 diabetes mellitus, two had chronic liver disease and one patient had leukaemia. Out of six patients with E. coli pyomyositis, two (33\%) died. AFB staining and mycobacterial culture of discharge was positive in two patients. Blood culture yielded the positive result in eight patients ( $n=8 / 62,12.90 \%$ ). Three patients had MSSA bacteremia: two had grown E. coli, and one patient each had MRSA, enterococci, and Acenitobacter bacteremia. Various factors including demographic profile, underlying medical diseases, clinical and laboratory parameters, length of hospital stay, and in-hospital outcome were compared between patients with pyomyositis caused by gram-positive and gram-negative organisms. None of them was statistically significantly different except patients with underlying medical diseases had significantly higher culture positivity with gram-negative organisms. Age at presentation was lower in patients with pyomyositis caused by gram-positive organisms although it did not reach statistical significance. We also did a detailed analysis and compared various parameters between patients with MSSA and MRSA infection. Two patients with polymicrobial (both gram-positive and gram-negative organisms) growth documented in drained pus were excluded from this analysis. Patients with MRSA pyomyositis presented relatively early in disease course although difference in duration of fever and muscle swelling before presentation did not reach statistical significance. Substantially higher number of patients with MRSA pyomyositis had received initial inappropriate antibiotic therapy. We also noticed trend towards longer hospital stay in patients with MRSA pyomyositis but it did not reach statistical significance. Further details of comparison between various parameters among patients with MRSA and MSSA pyomyositis have been elaborated in Table 4. Similarly, comparison of variables among patients with positive and negative pus culture result was done (Table 5). Serum urea was significantly higher in culture-positive patients. ESR and TLC were higher and haemoglobin level was lower in patients with positive pus culture report, though difference was not statistically significant. Various complications were noted in the significantly higher number of patients with positive culture result (32/38 [84.21\%] vs. 10/18 [55.56\%], $p=0.044$ ). Patients with positive culture report had significantly longer hospital stay (duration: median 15 days [IQR, 9.75 to 25] vs. 10.50 days [IQR, 5.75 to 14.50], $p=0.005$ ).

\section{Treatment, outcome, and factors associated with mortality}

Six patients (9.68\%) were managed with only intravenous antibiotics. Percutaneous drainage in the form of pigtail along with antibiotics was employed in 18 patients (32.14\%) and antibiotics plus surgical drainage was used in 38 cases $(67.86 \%)$. There was no difference in mortality among the group of patients managed with pigtail versus surgical drainage. Thirty-eight patients had organisms isolated from initial pus culture, and antibiotic sensitivity report was available. Among these patients with positive pus culture report, 25 received initial appropriate antibiotics and 13 remaining patients received inappropriate antibiotics. Out of 13 patients receiving initial inappropriate antibiotics, 10 patients developed features of sepsis, and five progressed to severe sepsis. Three of them died.

Six patients (9.68\%) had in-hospital mortality due to complications considered related to primary pyomyositis. During the hospital stay, 39 patients (62.9\%) were noted to have clinical features suggestive of sepsis. Twenty-two patients (35.48\%) progressed to the stage of severe sepsis. Table 6 shows the comparison of variables between survivors and non-survivors. Lower first-day serum albumin, initial inappropriate antibiotics, and advanced form of the disease (late stage) at presentation were associated with increased in-hospital mortality. Non-survivors had shorter hospital stay (duration: median 6.00 days [IQR, 2.75 to 7.50] vs. 13.91 days [IQR, 9.00 to 22.75 ], $p=0.001$ ). 
Table 4. Comparison of demographic data, clinical characteristics, laboratory features, hospital stay, and outcome among patients with MSSA or MRSA growth in pus culture in cohort of 24 pyomyositis patients

\begin{tabular}{|c|c|c|c|}
\hline Characteristic & $\begin{array}{l}\text { Patients with MSSA growth } \\
\text { in pus culture }(\mathrm{n}=17)\end{array}$ & $\begin{array}{l}\text { Patients with MRSA growth } \\
\text { in pus culture }(\mathrm{n}=7)\end{array}$ & $p$ value \\
\hline Age, yr & $22(16.50-27.50)$ & $17(15-50)$ & 0.710 \\
\hline Sex & & & 0.664 \\
\hline Male & $15(88.23)$ & $6(85.70)$ & \\
\hline Female & 2 (11.80) & 1 (14.29) & \\
\hline History of trauma & $3(17.64)$ & $2(28.57)$ & 0.531 \\
\hline \multicolumn{4}{|l|}{ Clinical features } \\
\hline Muscle pain & $17(100)$ & $7(100)$ & - \\
\hline Fever & $16(94.12)$ & $6(85.70)$ & 0.507 \\
\hline Back pain & $2(11.76)$ & $1(14.29)$ & 0.729 \\
\hline Swelling & $15(88.23)$ & $6(85.71)$ & 0.843 \\
\hline Redness & $6(35.29)$ & $3(42.86)$ & 0.562 \\
\hline Fever, day & $12(6.25-20.00)$ & $5.50\left(3.75^{-12.50}\right)$ & 0.065 \\
\hline Muscle swelling, day & $10(6.75-20.00)$ & $6.50(5.25-14.50)$ & 0.239 \\
\hline Pulse rate, /min & $100(88-109)$ & $98(96-110)$ & 0.576 \\
\hline $\mathrm{SBP}, \mathrm{mmHg}$ & $120(110-120)$ & $110(109-118)$ & 0.455 \\
\hline $\mathrm{DBP}, \mathrm{mmHg}$ & $80(70-80)$ & $70(60-80)$ & 0.087 \\
\hline Respiratory rate, /min & $20(16-21)$ & $19(14-32)$ & 0.710 \\
\hline GCS & $15(15-15)$ & $15(15-15)$ & 0.628 \\
\hline \multicolumn{4}{|l|}{ Laboratory parameter (first day) } \\
\hline Hemoglobin, g/dL & $10.30(8.80-12.25)$ & $8.70(8.00-8.90)$ & 0.087 \\
\hline $\mathrm{TLC}, / \mu \mathrm{L}$ & $14,300(9,100-18,200)$ & $10,300(8,000-17,600)$ & 0.455 \\
\hline $\mathrm{ESR}, \mathrm{mm} / \mathrm{hr}$ & $60(51-70)$ & $55(41-69)$ & 0.693 \\
\hline Serum urea, mg/dL & $44.50(24.00-68.50)$ & $47.00(17.00-140.00)$ & 0.804 \\
\hline Serum creatinine, $\mathrm{mg} / \mathrm{dL}$ & $0.90\left(0.55^{-1.25}\right)$ & $0.98(0.60-2.10)$ & 0.576 \\
\hline Total protein, g/dL & $5.93(5 \cdot 35-7.40)$ & $6.60(5.50-7.80)$ & 0.315 \\
\hline Serum albumin, g/dL & $2.50(2.25-2.95)$ & $2.70(2.10-3.20)$ & 0.901 \\
\hline SGOT, U/L & $61.00(32.50-82.00)$ & $49.00(38.00-60.25)$ & 0.664 \\
\hline SGPT, U/L & $56.00(31.50-93.00)$ & $33.00(30.50-45.00)$ & 0.130 \\
\hline Bilirubin, total, mg/dL & $0.80(0.65-1.05)$ & $1.00(0.80-1.40)$ & 0.209 \\
\hline Late stage at presentation & $3(17.65)$ & $1(14.29)$ & 0.712 \\
\hline Positive blood culture & $1(5.88)$ & 0 & 0.978 \\
\hline Initial inappropriate antibiotic & $1(5.88)$ & $5(71.42)$ & 0.003 \\
\hline \multicolumn{4}{|l|}{ Management } \\
\hline Percutaneous & $5(29.41)$ & $2(28.57)$ & 0.682 \\
\hline Surgical drainage & $12(70.59)$ & $5(71.43)$ & 0.605 \\
\hline Complications & $10(58.82)$ & $5(71.43)$ & 0.529 \\
\hline Hospital stay, day & $15(12-23)$ & $23(12-29)$ & 0.413 \\
\hline In-hospital mortality & $1(5.88)$ & $1(14.29)$ & 0.507 \\
\hline
\end{tabular}

Values are presented as median (interquartile range) or number (\%).

MSSA, methicillin-sensitive Staphylococcus aureus; MRSA, methicillin-resistant Staphylococcus aureus; SBP, systolic blood pressure; DBP, diastolic blood pressure; GCS, Glasgow coma scale; TLC, total leucocyte count; ESR, erythrocyte sedimentation rate; SGOT, serum glutamic oxaloacetic transaminase; SGPT, serum glutamic pyruvic transaminase. 
Table 5. Comparison of demographic data, clinical characteristics, laboratory features, hospital stay, and outcome among patients with positive or negative pus culture result in cohort of ${ }_{5} 6$ pyomyositis patients

\begin{tabular}{|c|c|c|c|}
\hline Characteristic & $\begin{array}{l}\text { Pus culture positive } \\
\qquad(\mathrm{n}=38)\end{array}$ & $\begin{array}{l}\text { Pus culture negative } \\
\qquad(\mathrm{n}=18)\end{array}$ & $p$ value \\
\hline Age, yr & $23.50(15-37.75)$ & $27(18.50-42)$ & 0.312 \\
\hline Sex & & & 0.136 \\
\hline Male & $31(81.58)$ & $17(95.83)$ & \\
\hline Female & $7(18.42)$ & $1(4.17)$ & \\
\hline History of trauma & $10(26.32)$ & $6(33 \cdot 33)$ & 0.459 \\
\hline Underlying medical conditions & $9(23.68)$ & $3(16.67)$ & 0.732 \\
\hline \multicolumn{4}{|l|}{ Clinical features } \\
\hline Muscle pain & $38(100)$ & $18(100)$ & - \\
\hline Fever & $32(84.21)$ & $12(66.67)$ & 0.171 \\
\hline Back pain & $8(21.05)$ & $4(22.22)$ & 0.837 \\
\hline Swelling & $31(81.58)$ & $13(72.22)$ & 0.712 \\
\hline Redness & $14(36.84)$ & $6(33 \cdot 33)$ & 0.867 \\
\hline Fever, day & $10(5-20)$ & $8(5 \cdot 50-18)$ & 0.928 \\
\hline Muscle swelling, day & $10(6-14 \cdot 50)$ & $11(6-20)$ & 0.818 \\
\hline Pulse rate, $/ \mathrm{min}$ & $100(89-110)$ & $87(80-98$ & 0.090 \\
\hline $\mathrm{SBP}, \mathrm{mmHg}$ & $110(110-120)$ & $120(110-130)$ & 0.122 \\
\hline $\mathrm{DBP}, \mathrm{mmHg}$ & $78(70-80)$ & $80(70-80)$ & 0.519 \\
\hline Respiratory rate, /min & $19(16-28)$ & $18(16-22)$ & 0.237 \\
\hline GCS & $15(15-15)$ & $15(15-15)$ & 0.604 \\
\hline \multicolumn{4}{|l|}{ Laboratory parameter (first day) } \\
\hline Hemoglobin, g/dL & $8.90(7.98-10.45)$ & $10.70(9.38-11.83)$ & 0.070 \\
\hline $\mathrm{TLC}, / \mu \mathrm{L}$ & $13,200(8,900-17,925)$ & $10,400(7,725-12,475)$ & 0.166 \\
\hline $\mathrm{ESR}, \mathrm{mm} / \mathrm{hr}$ & $56(43-66)$ & $50(25-65)$ & 0.330 \\
\hline Serum urea, mg/dL & $58.50(23.50-84.00)$ & $27.50(22.75-58.50)$ & 0.045 \\
\hline Serum creatinine, mg/dL & $0.90(0.50-1.23)$ & $0.80(0.50-1.00)$ & 0.384 \\
\hline Total protein, g/dL & $6.40(5.40-7.45)$ & $6.40(5.70-7.70)$ & 0.776 \\
\hline Serum albumin, g/dL & $2.60(2.15-2.95)$ & $2.70(2.20-3.60)$ & 0.751 \\
\hline SGOT, U/L & $49(35 \cdot 50-77 \cdot 50)$ & $40(25-80)$ & 0.272 \\
\hline SGPT, U/L & $59(29 \cdot 50-74.50)$ & $50(24-65)$ & 0.493 \\
\hline Bilirubin, total, mg/dL & $0.89\left(0.45^{-1.00}\right)$ & $0.80(0.40-1.00)$ & 0.297 \\
\hline Late stage at presentation & $6(15 \cdot 79)$ & $1(5 \cdot 56)$ & 0.367 \\
\hline Positive blood culture & $5(13.16)$ & $3(16.67)$ & 0.854 \\
\hline \multicolumn{4}{|l|}{ Management } \\
\hline Percutaneous & $10(26.32)$ & $8(44 \cdot 44)$ & 0.370 \\
\hline Surgical drainage & $28(73.68)$ & $10(55 \cdot 56)$ & 0.378 \\
\hline Complications & $32(84.2)$ & $10(55 \cdot 56)$ & 0.044 \\
\hline Hospital stay, day & $15(9.75-25)$ & $10.50(5.75-14.50)$ & 0.005 \\
\hline In-hospital mortality & $4(10.52)$ & $2(11.11)$ & 0.637 \\
\hline
\end{tabular}

Values are presented as median (interquartile range) or number (\%).

SBP, systolic blood pressure; DBP, diastolic blood pressure; GCS, Glasgow coma scale; TLC, total leucocyte count; ESR, erythrocyte sedimentation rate; SGOT, serum glutamic oxaloacetic transaminase; SGPT, serum glutamic pyruvic transaminase. 
Table 6. Comparison of demographic data, clinical characteristics, laboratory features, and hospital stay among survivors and non-survivors in cohort of 62 pyomyositis patients

\begin{tabular}{|c|c|c|c|}
\hline Characteristic & Survivors $(n=56)$ & Non-survivors $(n=6)$ & $p$ value \\
\hline Age, yr & $24.83(17.25-36.50)$ & $36.50(29-45)$ & 0.084 \\
\hline Sex & & & 0.168 \\
\hline Male & $50(89 \cdot 3)$ & $4(66.7)$ & \\
\hline Female & $6(10.7)$ & $2(33 \cdot 3)$ & \\
\hline Predisposing medical disorder & $10(17.9)$ & $2(33 \cdot 3)$ & 0.328 \\
\hline \multicolumn{4}{|l|}{ Clinical features } \\
\hline Muscle pain & $56(100)$ & $6(100)$ & - \\
\hline Fever & $43(76.8)$ & $5(83 \cdot 3)$ & 1.000 \\
\hline Back pain & $12(21.43)$ & $2(33 \cdot 33)$ & 0.500 \\
\hline Swelling & $41(73.21)$ & $6(100)$ & 0.105 \\
\hline Fever, day & $11(5.29-20.00)$ & $10(6.50-17.00)$ & 0.911 \\
\hline Muscle swelling, day & $10(6-20)$ & $14.50(7.75-18.50)$ & 0.543 \\
\hline Pulse rate, /min & $95.50(85.14-108.29)$ & $98(79-108.50)$ & 0.880 \\
\hline $\mathrm{SBP}, \mathrm{mmHg}$ & $116(109-123)$ & $115(107.50-147.50)$ & 0.972 \\
\hline $\mathrm{DBP}, \mathrm{mmHg}$ & $78(69-81)$ & $75(70-90)$ & 0.825 \\
\hline Respiratory rrate, /min & $18.29(16.00-22.00)$ & $22(19-31)$ & 0.067 \\
\hline GCS & $15(15-15)$ & $15\left(8.75^{-15.00)}\right.$ & 0.191 \\
\hline \multicolumn{4}{|l|}{ Laboratory parameter (first day) } \\
\hline Hemoglobin, g/dL & $9.53(8.25-11.18)$ & $9.15(7.98-10.38)$ & 0.662 \\
\hline $\mathrm{TLC}, / \mu \mathrm{L}$ & $11,933(8,375-17,650)$ & $9,000(4,075-10,750)$ & 0.053 \\
\hline $\mathrm{ESR}, \mathrm{mm} / \mathrm{hr}$ & $56.75(41.75-65.00)$ & $44(39-59)$ & 0.392 \\
\hline Serum urea, mg/dL & $34(22.20-68)$ & $56(27.50-157.25)$ & 0.218 \\
\hline Serum creatinine, $\mathrm{mg} / \mathrm{dL}$ & $0.83(0.56-1.18)$ & $0.84(0.40-1.88)$ & 0.933 \\
\hline Total protein, g/dL & $6.48(5.48-7.58)$ & $6.15(5 \cdot 50-6.58)$ & 0.279 \\
\hline Serum albumin, g/dL & $2.64(2.27-3.05)$ & $2.10(1.95-2.10)$ & 0.009 \\
\hline SGOT, U/L & $43(29-76)$ & $77.50(56.00-129.00)$ & 0.089 \\
\hline SGPT, U/L & $36.40(23.50-65.67)$ & $45.00(41.75-55.00)$ & 0.549 \\
\hline Bilirubin, total, mg/dL & $0.70(0.42-0.98)$ & $0.90\left(0.45^{-1.25}\right)$ & 0.437 \\
\hline Pus culture positive & $34 / 50(68)$ & $4 / 6(66.7)$ & 0.776 \\
\hline Gram-positive & $24 / 34(70.59)$ & $3 / 4(75)$ & 0.553 \\
\hline Gram-negative & $13 / 34(38.24)$ & $1 / 4(25)$ & 0.424 \\
\hline Positive blood culture & $6 / 56(10.71)$ & $2 / 6(33 \cdot 3)$ & 0.062 \\
\hline Initially inappropriate antibiotic & $10 / 34(29.41)$ & $3 / 4(75)$ & 0.002 \\
\hline Stage at presentation, late stage & $2 / 56(3.6)$ & $4 / 6(66.7)$ & 0.001 \\
\hline Hospital stay, day & $13.91(9.00-22.75)$ & $6.00(2.75-7.50)$ & 0.001 \\
\hline
\end{tabular}

Values are presented as median (interquartile range) or number (\%).

SBP, systolic blood pressure; DBP, diastolic blood pressure; GCS, Glasgow coma scale; TLC, total leucocyte count; ESR, erythrocyte sedimentation rate; SGOT, serum glutamic oxaloacetic transaminase; SGPT, serum glutamic pyruvic transaminase. 


\section{DISCUSSION}

This observational cohort study re-emphasizes the fact that primary pyomyositis is not an uncommon disease condition. Myalgia, fever, anemia, leukocytosis, raised ESR, and hypoalbuminemia were found in a large number of patients. Patients with underlying medical diseases were older, had a higher rate of gram-negative bacterial infections and were more likely to receive initial inappropriate antibiotic therapy. Patients with positive pus culture report had longer hospital stay and the higher rate of complications as compared to patients with negative pus culture report. Lower first-day serum albumin, initial inappropriate antibiotic therapy and advanced form of the disease at presentation were associated with increased in-hospital mortality.

Pyomyositis is primary infection of striated muscles, usually presenting as an intramuscular abscess. Sometimes it may present as a diffuse inflammatory process without abscess formation. This infective condition is seen in all age groups, but maximum number of patients are reported in age group 10 to 40 years [1-3]. The mean age of our study cohort was 29.9 years. In a descriptive review, patients from temperate regions were older (mean age, 34 years) as compared to cases reported from tropical countries (mean age, 28 years) [10]. This observation to some extent could be due to the higher number of patients with underlying predisposing conditions developing this infective disease in temperate areas. Studies reported both from tropical and temperate countries found patients with underlying medical disease conditions were older as was the case in index study as well $[5,10,15]$. Male preponderance noted in this study is reported previously in various studies as well [1-3].

The pathogenesis of this infective disease process is not precisely known till date. Healthy striated muscle is considered intrinsically resistant to bacterial infections as iron required for bacterial proliferation is sequestered by myoglobin. Local muscle tissue structure is altered by trauma, obvious or many times unrecognized, releasing sequestered iron from myoglobin. Abundant iron available after injury provides a suitable milieu resulting in rapid growth and proliferation of organisms implanted in damaged muscles from a bacteremic episode $[1-3,6]$. Various studies have documented history of trauma to the affected muscles in $5 \%$ to $50 \%$ of patients [1-13]. In our study as well only 16 patients $(25.8 \%)$ gave the history of trauma to affected muscles, so other unknown factors or occult muscle injury/damage must also exist. Several other mechanisms postulated in the pathogenesis of pyomyositis include nutritional deficiencies, viral and parasitic infections, lack of immunity against Staphylococcus, although many of these are not fully substantiated $[1-3,6]$. Pyomyositis was initially thought to be confined to tropical areas predominantly affecting young and healthy populations with no underlying medical comorbidities. In one of the largest series of 205 cases of 112 patients reported from Nigeria, none of the patients had underlying medical illness [6]. On the other hand, a descriptive review of patients over two decades from USA showed 119 patients (48\%) with underlying medical conditions, including diabetes, malignancy, chronic liver disease, and hematologic and rheumatologic conditions [10]. Twelve patients (19.35\%) had underlying medical diseases in our index study. Out of 155 patients diagnosed with pyomyositis over two decades in our institution including present study, 55 (35.48\%) had underlying medical disorders [3,4]. Differences in clinicoepidemiologic profile seen previously in patients of pyomyositis from temperate and tropical regions is thus getting blurred, which is getting reflected even in disease nomenclature with suggestions being made to rename this entity more appropriately as primary pyomyositis instead of tropical or temperate pyomyositis.

Early diagnosis of pyomyositis becomes difficult due to its relative unfamiliarity among physicians, non-specific early clinical symptoms and signs; and often lead to misdiagnosis. There are three discrete stages in the natural history of primary pyomyositis. This disease progresses from initial diffuse inflammation or invasive stage to focal abscess formation (suppurative stage) to late stage of dissemination and organ dysfunction [13,6]. The majority of patients in index study presented in the suppurative stage similar to previous studies [1-10]. Muscle pain was seen universally in all patients, but importantly fever was noted in 48 patients (77.4\%), similar to two studies reported from the same institution $[4,5]$. Leucocytosis with the shift to left and raised ESR was observed in the majority of our patients, as reported in previous studies [1-13]. These laboratory parameters with only muscle pain must prompt the clinician to keep py- 
omyositis as one of diagnostic possibility. We noticed higher level of mean ESR and TLC across three stages of illness at presentation, highest documented in the disseminated stage of infection. Mean serum protein and albumin at admission also showed graded relationship being lowest in the disseminated stage. ESR and TLC are the non-specific markers of acute inflammation while serum albumin is negative acute phase reactant [16]. Patients with infection/inflammation can have low serum albumin level. Various pathogenetic pathways include the decrease in albumin production, accelerated catabolism and increased permeability of the microvasculature leading to the excessive transcapillary passage of albumin to interstitial space. There is also diversion of synthetic pathway to other proteins of acute phase response [17-19]. These infection/inflammation induced mechanisms probably led to hypoalbuminemia in index study as well.

Most cases of primary pyomyositis are due to $S$. aureus. It is the causative agent in the majority of cases, accounting for up to $90 \%$ of infections reported from the tropics and up to $60 \%$ to $70 \%$ of cases reported from temperate areas [1-3]. Our study also showed the predominance of $S$. aureus as the causative agent. Out of 27 patients with growth of gram-positive organisms in drained pus, MSSA was isolated in 18 and MRSA in eight patients. Resistance to methicillin, a penicillinase-stable $\beta$-lactam was first noticed among S. aureus in the United Kingdom in 1961 [20]. These strains are multidrug resistant. Cases of infections caused by a different MRSA strains, CA-MRSA, infecting apparently healthy individuals without any healthcare contact, harbouring smaller and more mobile staphylococcal cassette-chromosome types (IV and V) and susceptible to many non- $\beta$-lactam antimicrobial drugs were reported from USA in $1990 \mathrm{~s}$ initially [21,22]. These MRSA strains since then have spread throughout world causing variety of infections. There are case reports and series reporting pyomyositis caused by both of these strains in medical literature. But differentiation between healthcare-associated MRSA (HA-MRSA) and CA-MRSA strains based on genetic markers and virulence factors is getting blurred lately with increasing number of isolates showing characteristics of both HA-MRSA and CA-MRSA pathogens [2325]. This could possibly be due to exchange of genetic materials between these strains. We also documented eight cases of pyomyositis caused by MRSA strain. Significantly higher number of MRSA related pyomyositis patients received in-appropriate antibiotic therapy initially. There was no difference in hospital mortality. There was although trend towards longer hospital stay in patients with MRSA pyomyositis. In a retrospective case series of 39 adult patients with pyomyositis, oxacillin-resistant $S$. aureus comprised 25\% (8/32) of infections due to $S$. aureus. They found increasing number of cases of oxacillin-resistance during study from one of $18 \mathrm{~S}$. aureus isolates from 1994 to 2004 to seven of 14 isolates from 2004 to 2006 [26]. In our institute as well, studies have shown increasing number of MRSA isolates from none during 1992 to 1999 to four during study period of 7 and half years from 2000 to June 2007 from pus culture $[4,5]$. In the index study of 6 and half years duration, eight MRSA isolates were reported from drained pus. Number of studies have tried to discern impact of methicillin-resistance on hospital outcomes including mortality rates among patients infected with $S$. aureus. These studies yielded variable results. A single-center prospective study by Melzer et al. [27] showed that MRSA infection was not associated with increased mortality. Similar result was also reported in a study by Wolkewitz et al. [28]. Number of studies on the other hand, reported hospital mortality rate significantly higher in infections caused by MRSA strain. As patients with MRSA infection are more likely than patients with MSSA infection to be older, to have more comorbid illnesses and greater severity of illness; inadequate control of these confounding factors remained a major concern. An observational study involving cohort of 500 patients with MSSA bacteraemia, 111 patients with CA-MRSA, and 133 patients with HA-MRSA bacteraemia evaluated the impact of the MRSA strain on mortality in patients with CA-MRSA or HA-MRSA bacteraemia by comparison with mortality in patients with MSSA bacteraemia. This study concluded that infection with traditional hospital strain of MRSA was associated with a significantly higher mortality rate than infection with MSSA, independent of the confounding effect from underlying disease and acute severity of bacteraemia [29]. Cosgrove et al. [30] examined data from 31 cohort studies including 3,963 patients (34\% of whom were infected with methicillin-resistant strains) in a meta-analysis. It showed that bacteraemia due to MRSA is associated with increased mortality compared with 
MSSA bacteraemia. This finding remained significant after taking care of confounding variables [30]. As stated earlier, we did not find significant difference in duration of hospital stay and hospital mortality rate among MSSA and MRSA infected patients with pyomyositis. This result could be due to the small numbers of MSSA and MRSA pyomyositis cases in present study. Pyomyositis is usually caused by gram-positive organisms but lately increasing number of cases caused be gram-negative organisms are being reported. We also encountered 14 patients with pyomyositis caused by gram-negative organisms. In a retrospective observational study by Chiu et al. [15], 35 cases of primary pyomyositis were identified during the study period and 23 of them had underlying medical diseases. Gram-negative organisms were isolated in seven patients (30.4\%) with underlying diseases. They found that patients who suffered from primary pyomyositis with underlying diseases were older, had a higher rate of gram-negative bacterial infections, bacteraemia, and hospital mortality [15]. Similar result was reported in a study from Indian subcontinent as well [5]. The index study also reported that patients with comorbidities were older, had higher culture positivity with gram-negative organisms. Importantly, higher number of these patients received inappropriate antibiotics initially. E. coli was the most common pathogenic organism isolated among patients with pyomyositis caused by gram-negative organisms. There are few case reports and small case series of E. coli pyomyositis. Vigil et al. [31] in a retrospective observational study analysed six cases of $E$. coli pyomyositis in patients with hematologic malignancy. Two (33\%) died, despite receiving carbapenem therapy. All E. coli isolates were quinolone resistant; $55 \%$ produced an ESBL [31]. We found four E. coli isolates to be resistant to quinolone. Two patients (33\%) with E. coli pyomyositis in index study died. Zalavras et al. [26] retrospectively reviewed records of 39 adult patients with diabetes mellitus treated for thigh pyomyositis. Their mean age was 45 years. Gram-negative organisms were cultured in $14 \%$ (6/39) of patients and three grew E. coli [26]. In a retrospective descriptive review of human immunodeficiency virus (HIV)-negative patients with underlying medical conditions and pyomyositis, $14.3 \%$ (17/119) patients had gram-negative organisms as infecting pathogen [10]. These findings emphasize the fact that antibiotic coverage for gram-negative organisms should be empirically initiated in patients of pyomyositis with concurrent medical disorders/immunosuppressive conditions. Interestingly, we found patients with positive pus culture report had longer hospital stay and the higher rate of complications as compared to patients with negative pus culture report. A retrospective observational study of 24 children reported from Taiwan also showed similar results [32]. The plausible explanation could be greater load of micro-organisms leading to more marked inflammatory response which was also reflected in higher mean TLC, ESR, and lower mean serum albumin in culture positive patients, resulting in higher rate of complications. Other possible explanation could be faster reduction in load of micro-organisms from pus with appropriate antibiotics in culture negative patients resulting in lesser complications, resulting understandably in the shorter hospital stay.

The most common site of involvement was thigh muscles, followed by iliopsoas. Previous studies irrespective of the region have also noted that pyomyositis usually involves the largest muscle groups located around the pelvic girdle and lower extremities [1-10]. These observations further strengthen the postulation that greater degree of movement in muscle groups located around the pelvic girdle and lower extremities may cause subclinical trauma to these muscles making them more susceptible to infection by bacteria from a subsequent bacteremic episode.

The stage at presentation decides treatment options for patients with pyomyositis. Oral or intravenous antibiotics alone would suffice during the early stage of the infection, the diffuse inflammatory stage. Abscess formation, however, requires antibiotic with appropriate drainage procedure. There was no difference in mortality based on methods of drainage of pus. Six patients (9.68\%) had in-hospital mortality due to complications considered related to primary pyomyositis. Lower firstday serum albumin, initial inappropriate antibiotic therapy and advanced form of the disease (late stage) at presentation were associated with increased in-hospital mortality. Serum albumin plays diverse, complex, and important roles in maintaining physiologic homeostasis [18]. Hypoalbuminemia may either directly contribute to poor outcomes, or it could merely be a marker for other "upstream" pathologic processes such as malnutrition or infection/inflammation. It appears to be a 
reliable prognostic indicator in various contexts [18,33]. It was found to be a potent, dose-dependent independent predictor of poor outcome in a meta-analysis of 90 cohort studies with 291,433 patients. The association between hypoalbuminemia and poor outcome appeared to be independent of both nutritional status and inflammation [34]. As stated earlier, inflammation induced by infective condition predominantly contributed to hypoalbuminemic state in our study. Initial inappropriate antibiotic therapy has been documented in studies to lead to the poorer outcome. The advanced form of the disease at presentation also would understandably have the poorer prognosis. These patients also had the greater degree of inflammation leading to greater lowering of serum albumin.

In conclusion, this study reemphasizes the fact that primary pyomyositis is not an uncommon disease entity. Patients with underlying medical diseases were older, had a higher rate of gram-negative bacterial infections and were more likely to receive initial inappropriate antibiotic therapy. Patients with positive pus culture report had longer hospital stay and the higher rate of complications. Lower first-day serum albumin, initial inappropriate antibiotic therapy and advanced form of the disease at presentation were associated with increased in-hospital mortality.

\section{KEY MESSAGE}

1. Primary pyomyositis is not an uncommon infective disease entity. Differences in clinicoepidemiologic profile seen previously in these patients from temperate and tropical regions is getting increasingly blurred, which is reflected in disease nomenclature as well.

2. Patients with comorbidities should initially receive empirical antibiotics effective for gram negative organisms besides coverage for usual gram positive pathogens.

3. Primary pyomyositis patients with positive pus culture and hypoalbuminemia should be aggressively managed as these patients were more likely to have higher hospital morbidity and mortality.

\section{Conflict of interest}

No potential conflict of interest relevant to this article was reported.

\section{REFERENCES}

1. Bickels J, Ben-Sira L, Kessler A, Wientroub S. Primary pyomyositis. J Bone Joint Surg Am 2002;84-A:2277-2286.

2. Chauhan S, Jain S, Varma S, Chauhan SS. Tropical pyomyositis (myositis tropicans): current perspective. Postgrad Med J 2004;80:267-270.

3. Agarwal V, Chauhan S, Gupta RK. Pyomyositis. Neuroimaging Clin N Am 2011;21:975-983.

4. Malhotra P, Singh S, Sud A, Kumari S. Tropical pyomyositis: experience of a tertiary care hospital in north-west India. J Assoc Physicians India 2000;48:1057-1059.

5. Sharma A, Kumar S, Wanchu A, et al. Clinical characteristics and predictors of mortality in 67 patients with primary pyomyositis: a study from North India. Clin Rheumatol 2010;29:45-51.

6. Chiedozi LC. Pyomyositis: review of 205 cases in 112 patients. Am J Surg 1979;137:255-259.

7. Gambhir IS, Singh DS, Gupta SS, Gupta PR, Kumar M. Tropical pyomyositis in India: a clinico-histopathological study. J Trop Med Hyg 1992;95:42-46.

8. Chattopadhyay B, Mukhopadhyay M, Chatterjee A, Biswas PK, Chatterjee N, Debnath NB. Tropical pyomyositis. N Am J Med Sci 2013;5:600-603.

9. Martinez-de Jesus FR, Mendiola-Segura I. Clinical stage, age and treatment in tropical pyomyositis: a retrospective study including forty cases. Arch Med Res 1996;27:165-170.

10. Crum NF. Bacterial pyomyositis in the United States. Am J Med 2004;117:420-428.

11. Hall RL, Callaghan JJ, Moloney E, Martinez S, Harrelson JM. Pyomyositis in a temperate climate: presentation, diagnosis, and treatment. J Bone Joint Surg Am 1990;72:1240-1244.

12. Unnikrishnan PN, Perry DC, George H, Bassi R, Bruce CE. Tropical primary pyomyositis in children of the UK: an emerging medical challenge. Int Orthop 2010;34:109113.

13. Block AA, Marshall C, Ratcliffe A, Athan E. Staphylococcal pyomyositis in a temperate region: epidemiology and modern management. Med J Aust 2008;189:323-325.

14. Levy MM, Fink MP, Marshall JC, et al. 2001 SCCM/ES- 
ICM/ACCP/ATS/SIS international sepsis definitions conference. Crit Care Med 2003;31:1250-1256.

15. Chiu SK, Lin JC, Wang NC, Peng MY, Chang FY. Impact of underlying diseases on the clinical characteristics and outcome of primary pyomyositis. J Microbiol Immunol Infect 2008;41:286-293.

16. Markanday A. Acute phase reactants in infections: evidence-based review and a guide for clinicians. Open Forum Infect Dis 2015;2:ofvo98.

17. Don BR, Kaysen G. Serum albumin: relationship to inflammation and nutrition. Semin Dial 2004;17:432-437.

18. Nicholson JP, Wolmarans MR, Park GR. The role of albumin in critical illness. Br J Anaesth 2000;85:599-610.

19. Fleck A, Raines G, Hawker F, et al. Increased vascular permeability: a major cause of hypoalbuminaemia in disease and injury. Lancet 1985;1:781-784.

20. Jevons MP. “Celbenin": resistant staphylococci. Brit Med J 1961;1:124-125.

21. Saravolatz LD, Markowitz N, Arking L, Pohlod D, Fisher E. Methicillin-resistant Staphylococcus aureus: epidemiologic observations during a community-acquired outbreak. Ann Intern Med 1982;96:11-16.

22. Herold BC, Immergluck LC, Maranan MC, et al. Community-acquired methicillin-resistant Staphylococcus aureus in children with no identified predisposing risk. JAMA 1998;279:593-598.

23. Popovich KJ, Weinstein RA, Hota B. Are community-associated methicillin-resistant Staphylococcus aureus (MRSA) strains replacing traditional nosocomial MRSA strains? Clin Infect Dis 2008;46:787-794.

24. Saiman L, O'Keefe M, Graham PL 3rd, et al. Hospital transmission of community-acquired methicillin-resistant Staphylococcus aureus among postpartum women. Clin Infect Dis 2003;37:1313-1319.

25. Huang H, Flynn NM, King JH, Monchaud C, Morita $\mathrm{M}$, Cohen SH. Comparisons of community-associated methicillin-resistant Staphylococcus aureus (MRSA) and hospital-associated MSRA infections in Sacramento, Cal- ifornia. J Clin Microbiol 2006;44:2423-2427.

26. Zalavras CG, Rigopoulos N, Poultsides L, Patzakis MJ. Increased oxacillin resistance in thigh pyomyositis in diabetic patients. Clin Orthop Relat Res 2008;466:1405-1409.

27. Melzer M, Eykyn SJ, Gransden WR, Chinn S. Is methicillin-resistant Staphylococcus aureus more virulent than methicillin-susceptible S. aureus? A comparative cohort study of British patients with nosocomial infection and bacteremia. Clin Infect Dis 2003;37:1453-1460.

28. Wolkewitz M, Frank U, Philips G, Schumacher M, Davey P; BURDEN Study Group. Mortality associated with in-hospital bacteraemia caused by Staphylococcus aureus: a multistate analysis with follow-up beyond hospital discharge. J Antimicrob Chemother 2011;66:381-386.

29. Chen SY, Wang JT, Chen TH, et al. Impact of traditional hospital strain of methicillin-resistant Staphylococcus aureus (MRSA) and community strain of MRSA on mortality in patients with community-onset $\mathrm{S}$ aureus bacteremia. Medicine (Baltimore) 2010;89:285-294.

30. Cosgrove SE, Sakoulas G, Perencevich EN, Schwaber MJ, Karchmer AW, Carmeli Y. Comparison of mortality associated with methicillin-resistant and methicillin-susceptible Staphylococcus aureus bacteremia: a meta-analysis. Clin Infect Dis 2003;36:53-59.

31. Vigil KJ, Johnson JR, Johnston BD, et al. Escherichia coli pyomyositis: an emerging infectious disease among patients with hematologic malignancies. Clin Infect Dis 2010;50:374-380.

32. Chiu NC, Hsieh MC, Chi H, Huang FY. Clinical characteristics of pyomyositis in children: 20-year experience in a medical center in Taiwan. J Microbiol Immunol Infect 2009;42:494-499.

33. Goldwasser P, Feldman J. Association of serum albumin and mortality risk. J Clin Epidemiol 1997;50:693-703.

34. Vincent JL, Dubois MJ, Navickis RJ, Wilkes MM. Hypoalbuminemia in acute illness: is there a rationale for intervention? A meta-analysis of cohort studies and controlled trials. Ann Surg 2003;237:319-334 